\title{
The effect of genetic selection for Johne's disease resistance in dairy cattle: Results of a genetic-epidemiological model
}

\author{
K. J. E. van Hulzen, ${ }^{\star} \dagger^{1}$ A. P. Koets, ${ }^{\star}$ M. Nielen, ${ }^{*}$ H. C. M. Heuven, $\ddagger$ J. A. M. van Arendonk,§ \\ and D. Klinkenberg* \\ ${ }^{*}$ Department of Farm Animal Health, Utrecht University, PO Box 80.151, 3508 TD Utrecht, the Netherlands \\ †Department of Human Genetics, Radboud University Medical Center, PO Box 9101, 6500 HB Nijmegen, the Netherlands \\ $\mp$ Clinical Sciences of Companion Animals, Utrecht University, PO Box 80.163, 3508 TD Utrecht, the Netherlands \\ §Animal Breeding and Genomics Centre, Wageningen University, PO Box 338, $6700 \mathrm{AH}$ Wageningen, the Netherlands
}

\begin{abstract}
The objective of this study was to model genetic selection for Johne's disease resistance and to study the effect of different selection strategies on the prevalence in the dairy cattle population. In the Netherlands, a certification-and-surveillance program is in use to reduce prevalence and presence of sources of infection in milk by culling ELISA-positive dairy cows in infected herds. To investigate the additional genetic effect of this program, a genetic-epidemiological model was developed to assess the effect of selection of cows that test negative for Johne's disease (dam selection). The genetic effect of selection at the sire level was also considered (sire selection), assuming selection of $80 \%$ of sires producing the most resistant offspring based on their breeding values, as well as the combined effect. Parameters assumed to be affected by genetic selection were the length of the latent period, susceptibility (i.e., the number of infectious doses needed to become infected), or the length of susceptible period as a calf. The effect of selection was measured by the time in years required to eliminate infection. Sensitivity analysis was performed for heritability, accuracy of selection, and intensity of selection. For dam selection, responses to selection were small, requiring 379 to $702 \mathrm{yr}$ for elimination. For sire selection, responses were much larger, although elimination still required 147 to $223 \mathrm{yr}$. The response to selection was largest if genetic selection affected the length of the susceptible period, followed by the susceptibility, and finally the length of the latent period. Genetic selection for Johne's disease resistance by certification and surveillance is too slow for practical purpose, but that selection on the sire level is able to contribute to the control of Johne's disease in the long run.
\end{abstract}

Received May 16, 2013.

Accepted November 27, 2013.

${ }^{1}$ Corresponding author: kimmvanhulzen@gmail.com
Key words: Johne's disease, genetic-epidemiological model, selection response, dairy cow

\section{INTRODUCTION}

Mycobacterium avium subspecies paratuberculosis (MAP) is the causative agent of Johne's disease. Infection with Johne's disease is characterized by lesions in the distal part of the ileum, mainly seen in ruminants. Ileal lesions hinder an efficient nutrient uptake and cows suffering from Johne's disease show diarrhea, weight loss, and a decrease in milk production (Stabel, 1998).

Classical control strategies to eradicate MAP from infected dairy cattle farms are (1) management restrictions to reduce MAP transmission and (2) test and cull strategies to reduce the sources of infection. Management restrictions to reduce MAP transmission focus mainly on avoiding contact of susceptible young stock with infected animals by, for example, separation of calves from dams immediately after birth (Bastida and Juste, 2011). For test and cull strategies to reduce the sources of infection, an ELISA is most commonly used to identify infected cows. In the Netherlands, a certification-and-surveillance program aiming to reduce sources of infection uses an ELISA in milk to detect and cull infectious dairy cows in infected herds, once every year (Nielsen, 2009). The ELISA is cheap, highly specific, but of low sensitivity (Whitlock et al., 2000), so that even cows in advanced stages of disease may not be detected. Although management restrictions and test and cull strategies are able to reduce the rate of infection considerably, eradication of MAP has been shown to be difficult and additional approaches to control Johne's disease are needed. One additional approach could be genetic selection for cows resistant to Johne's disease.

Earlier research showed heritability estimates of susceptibility to Johne's disease in cattle ranging from 0.03 to 0.23 (Koets et al., 2000; Mortensen et al., 2004; Gonda et al., 2006; Hinger et al., 2007; Attalla et al., 
2010; van Hulzen et al., 2011; Küpper et al., 2012), where susceptibility to disease was defined as having MAP-specific antibodies in milk or serum, or MAPpositive fecal culture or tissue culture of MAP. Selection for resistance to an infectious disease by removing positive animals has a direct genetic effect by reducing the number of offspring from susceptible cows. In addition, it has an indirect epidemiological effect, because if animals are less susceptible, fewer animals will become infected, thus reducing total MAP in the environment and exposure to MAP for all animals. To be able to accurately predict the long-term effect of genetic selection for disease resistance, both effects should be taken into account, so it is necessary to combine the heritability estimates with an epidemiological model, as Nieuwhof et al. (2009) did for foot rot in sheep.

The objective of this study was to model genetic selection for Johne's disease resistance and to study the effect on the prevalence in the dairy cattle population using a deterministic genetic-epidemiological model. Different breeding strategies were evaluated. In the first scenario on the selection of dams, the additional genetic effect of the certification-and-surveillance program currently used in the Netherlands was investigated (dam selection). The ELISA results as collected in the Dutch certification-and-surveillance program are used to select dams based on their genetic merit for resistance to Johne's disease as described above. In the second scenario on the selection of sires, the effect of selection to the male side of the breeding program was investigated (sire selection). In the third scenario, the effect of selection to the female side was combined with selection on the male side (combined selection).

\section{MATERIALS AND METHODS}

\section{Epidemiological Model}

The model was adapted from the model described by Marcé et al. (2011a) that was used to assess the effect of contact structure on MAP transmission in persistently infected dairy herds. The model was chosen because it includes the most up-to-date knowledge on the infection and an environmental compartment to model infection through contamination in the environment. For the purposes of this study, the model was simplified by reducing the multiple susceptible, transiently infected, and environmental compartments to one for each stage (Figure 1).

In our model, the host population is divided into susceptible animals $(S)$, transiently infected animals $(T)$, latently infected animals $(L)$, subclinically infected animals $\left(I_{S}\right)$, clinically infected animals $\left(I_{C}\right)$, and resistant animals due to aging $(R)$. Calves are born either sus- ceptible or transiently infected through vertical transmission. Susceptible animals can become transiently infected through environmental contamination, but if they do not become infected during early age (first 50 wk of life) they gain long-lasting resistance to infection due to physiological changes (age-dependent susceptibility). The presence and persistence of MAP in the environment is considered by modeling environmental contamination $(E)$. Calves $(T)$ and adult cows $\left(I_{S}\right.$ and $\left.I_{C}\right)$ are considered to be infectious (i.e., they shed MAP in their feces and contaminate the environment). The quantity of MAP organisms shed by an animal depends on its infection status (Marcé et al., 2011a). The $E$ is measured in units of MAP excreted on a weekly base by a clinically infected cow.

The equations describing the weekly rate of change (dt; in proportions) in each compartment are as follows:

$$
\begin{aligned}
& \mathrm{d} S / \mathrm{dt}=\varphi_{S}-\lambda S-\nu S-\mu S ; \\
& \mathrm{d} E / \mathrm{dt}=\psi_{T} T+\psi_{I_{S}} I_{S}+\psi_{I_{C}} I_{C}-\mu_{E} E ; \\
& \mathrm{d} T / \mathrm{dt}=\varphi_{T}+\lambda S-\gamma_{T} T-\mu T ; \\
& \mathrm{d} L / \mathrm{dt}=\gamma_{T} T-\gamma_{L} L-\mu L ; \\
& \mathrm{d} I_{S} / \mathrm{dt}=\gamma_{L} L-\gamma_{I_{S}} I_{S}-\mu I_{S} ; \\
& \mathrm{d} I_{C} / \mathrm{dt}=\gamma_{I_{S}} I_{S}-\mu_{I_{C}} I_{C} ; \\
& \mathrm{d} R / \mathrm{dt}=\nu S-\mu R,
\end{aligned}
$$

where $\varphi_{S}$ is the birth rate of susceptible animals, $\lambda$ is the rate at which susceptible animals become infected $(\lambda=\beta E$, where $\beta$ is the rate of transmission), $\nu$ is the rate at which susceptible animals become resistant, $\mu$ is the death rate, $\psi_{T}$ is the rate at which a transiently infectious animal sheds infectious doses in the environment, $\psi_{I_{S}}$ is the rate at which a subclinically infectious animal sheds infectious doses in the environment, $\psi_{I_{C}}$ is the rate at which a clinically infectious animal sheds infectious doses in the environment, $\mu_{E}$ is the outflow rate of infectious doses from the environment, $\varphi_{T}$ is the birth rate of transiently infected animals from latently and subclinically infectious cows, $\gamma_{T}$ is the rate at which transiently infectious animals become latently infected, $\gamma_{L}$ is the rate at which latently infected animals become subclinically infectious, $\gamma_{I_{S}}$ is the rate at which subclinically infectious animals become clinically infectious, and $\mu_{I_{C}}$ is the rate at which clinically infectious animals are removed from the farm. All parameters are also defined in Table 1. Parameter values used in the model were adapted from the literature (Marcé et al., $2011 \mathrm{a}, \mathrm{b})$, with the exception of $\beta$. The model was calibrated with $\beta$ to obtain a start prevalence for Johne's disease infection that reflected an average Dutch herd (0.087; van Hulzen et al., 2011). 


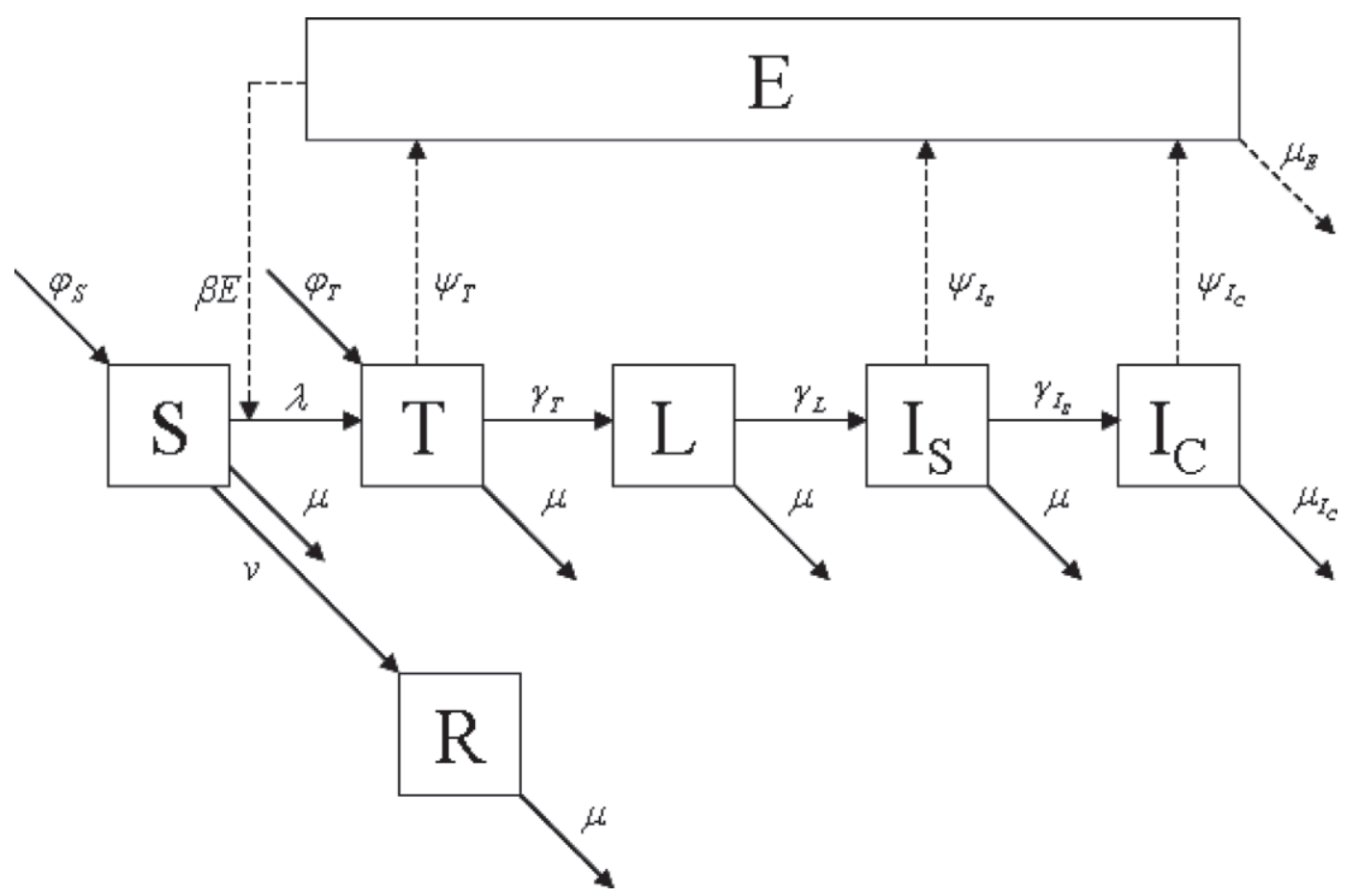

Figure 1. Schematic representation of the path of infection with Johne's disease, where $E$ is the environmental compartment and all other compartments represent disease states. $S=$ density of hosts susceptible to infection; $T=$ density of transiently infected hosts; $L=$ density of latently infected hosts; $I_{S}=$ density of subclinically infected hosts; $I_{C}=$ density of clinically infected hosts; $R=$ density of hosts resistant to infection; $\mu_{E}=$ outflow rate of infectious doses from the environment; $\varphi_{S}=$ birth rate of susceptible animals; $\beta=$ rate of transmission; $\varphi_{T}=$ birth rate of transiently infected animals from latently and subclinically infectious cows; $\psi_{T}=$ rate at which a transiently infectious animal sheds infectious doses in the environment; $\psi_{I_{S}}=$ rate at which a subclinically infectious animal sheds infectious doses in the environment; $\psi_{I_{C}}=$ rate at which a clinically infectious animal sheds infectious doses in the environment; $\lambda=$ rate at which susceptible animals become transiently infectious; $\gamma_{T}=$ rate at which transiently infectious animals become latently infected; $\gamma_{L}=$ rate at which latently infected animals become subclinically infectious; $\gamma_{I_{S}}=$ rate at which subclinically infectious animals become clinically infectious; $\mu=$ death rate; $\mu_{I_{C}}=$ rate at which clinically infectious animals are removed from the farm; $\nu=$ rate at which susceptible animals become resistant.

Losses from the Host Population. Losses occur as a result of routine culling and sales or deaths. These losses are summarized by parameter $\mu$, which is similar in all compartments except $I_{C}$. For clinically infected animals, losses from the herd are summarized by parameter $\mu_{I_{C}}$, which includes an increased loss due to additional culling related to clinical Johne's disease.

Births to the Host Population. Animals in the latently infected $(L)$, subclinically infected $\left(I_{S}\right)$, clinically infected $\left(I_{C}\right)$, and resistant $(R)$ compartments are assumed to give birth to newborn calves. Vertical transmission results in the birth of transiently infected calves, with a probability of 0.15 for latently and subclinically infected cows, and a probability of 0.65 for clinically infected cows (Benedictus et al., 2008; Whittington and Windsor, 2009). Resistant animals are assumed to give birth only to susceptible calves. The overall birth rate is equal to the overall loss rate to ensure a constant population size.

Equilibrium Prevalence. Because of our aim to select test-negative animals, we define prevalence as the proportion of test positives, which are all animals in states $I_{S}$ and $I_{C}$. An equilibrium state is reached when the environmental contamination as well as the proportion of animals in each compartment does not change. In other words, the rates of change for all compartments are all zero (i.e., $\mathrm{d} E / \mathrm{d} t=\mathrm{d} S / \mathrm{d} t=\mathrm{d} T / \mathrm{d} t=\mathrm{d} L /$ $\left.\mathrm{d} t=\mathrm{d} I_{S} / \mathrm{d} t=\mathrm{d} I_{C} / \mathrm{d} t=\mathrm{d} R / \mathrm{d} t=0\right)$. The equilibrium prevalence $p\left(\frac{I_{S}+I_{C}}{N}\right)$, where $N$ is the total number of cows, can be calculated as a function of all parameters included in the model, or a single parameter value can be calculated, given all other parameters and the equilibrium prevalence. For computational ease, this was programmed in Mathematica 7.0 software (Wolfram Research, Champaign, IL).

\section{Predicting Responses to Selection}

In this study, the epidemiological model described above was used to predict responses to selection (expressed as a reduction in prevalence of test positives) 
Table 1. Parameter definitions and values (all rates are per week)

\begin{tabular}{|c|c|c|c|}
\hline Parameter & Definition & Value & Source \\
\hline$S$ & Density of hosts susceptible to infection & - & - \\
\hline$T$ & Density of transiently infected hosts & - & - \\
\hline$L$ & Density of latently infected hosts & - & - \\
\hline$I_{S}$ & Density of subclinically infected hosts & - & - \\
\hline$I_{C}$ & Density of clinically infected hosts & - & - \\
\hline$R$ & Density of hosts resistant to infection & - & - \\
\hline E & Density of infectious doses in the environment & - & - \\
\hline$\varphi$ & Total birth rate & $\mu S+\mu T+\mu L+\mu I_{S}+\mu_{I_{C}} I_{C}+\mu R$ & \\
\hline$\varphi_{S}$ & Birth rate of susceptible animals & $\frac{0.85 L+0.85 I_{S}+0.35 I_{C}+R}{L+I_{S}+I_{C}+R} \varphi$ & Benedictus et al. (2008); Whittington and Windsor (2009) \\
\hline$\varphi_{T}$ & $\begin{array}{l}\text { Birth rate of transiently infected animals from latently and } \\
\text { subclinically infectious cows }\end{array}$ & $\frac{0.15 L+0.15 I_{S}+0.65 I_{C}}{L+I_{S}+I_{C}} \varphi$ & Benedictus et al. (2008); Whittington and Windsor (2009) \\
\hline$\lambda$ & $\begin{array}{l}\text { The rate at which susceptible animals become transiently } \\
\text { infectious }\end{array}$ & $\beta E$ & \\
\hline$\beta$ & The rate of transmission & 0.175 & \\
\hline$\gamma_{T}$ & $\begin{array}{l}\text { The rate at which transiently infectious animals become } \\
\text { latently infected }\end{array}$ & 0.04 & van Roermund et al. (2007) \\
\hline$\gamma_{L}$ & $\begin{array}{l}\text { The rate at which latently infected animals become } \\
\text { subclinically infectious }\end{array}$ & 0.02 & Nielsen and Ersbøll (2006); Nielsen (2008) \\
\hline$\gamma_{I_{S}}$ & $\begin{array}{l}\text { The rate at which subclinically infectious animals become } \\
\text { clinically infectious }\end{array}$ & 0.01 & Matthews (1947) \\
\hline$\nu$ & The rate at which susceptible animals become resistant & 0.02 & Marcé et al. (2011b) \\
\hline$\psi_{T}$ & $\begin{array}{l}\text { The rate at which a transiently infectious animal sheds } \\
\text { infectious doses in the environment }\end{array}$ & 0.00003 & van Roermund et al. (2007) \\
\hline$\psi_{I_{S}}$ & $\begin{array}{l}\text { The rate at which a subclinically infectious animal sheds } \\
\text { infectious doses in the environment }\end{array}$ & 0.00026 & Rossiter and Burhans (1996) \\
\hline$\psi_{I_{C}}$ & $\begin{array}{l}\text { The rate at which a clinically infectious animal sheds } \\
\text { infectious doses in the environment }\end{array}$ & 1 & Jørgensen (1982); Whittington et al. (2000) \\
\hline$\mu$ & Death rate & 0.005 & \\
\hline$\mu_{I_{C}}$ & $\begin{array}{l}\text { The rate at which clinically infectious animals are removed } \\
\text { from the farm }\end{array}$ & 0.04 & Marcé et al. (2011b) \\
\hline$\mu_{E}$ & Outflow rate of infectious doses from the environment & 0.40 & Jorgensen (1977); Whittington et al. (2004) \\
\hline
\end{tabular}


in the dairy cattle population for (1) genetic selection in cows (i.e., selection of those cows that test negative for Johne's disease at the time point at which the observations are made; dam selection), (2) selection of $80 \%$ of sires producing the least-susceptible offspring based on their breeding values (sire selection), and (3) selection based on a combination of 1 and 2 (combined selection).

Assumptions had to be made as to which epidemiological parameter was affected by genetic selection. Three parameters were separately considered: (1) the rate at which latently infected animals become infectious (i.e., selecting animals that have an increased period of latency; $\left.\gamma_{L}\right),(2)$ the transmission rate (i.e., selecting animals that are more resistant to infection, requiring exposure to a greater number of infectious doses before becoming infected; $\beta$ ), and (3) the rate at which susceptible animals become resistant (i.e., selecting animals that have a shorter period of susceptibility; $\nu)$. Responses in parameters were assessed independent of each other in separate analyses, as observed differences in prevalence were due to 1 of these 3 parameters. It was assumed that the inverse rates were normally distributed in the population, reflecting a normal distribution of the mean latent period $\left(1 / \gamma_{L}\right.$ weeks), the mean number of infectious doses needed to become infected $(1 / \beta$ infectious doses), and the mean age of becoming age resistant $(1 / \nu$ weeks) with constant underlying variances, thus ignoring the Bulmer effect (Hill, $2010)$. Start prevalence $\left(p_{0}\right)$ and heritability $\left(h^{2}\right)$ of resistance were $p_{0}=\frac{I_{S}+I_{C}}{N}=0.087$ and $h^{2}=0.10($ van Hulzen et al., 2011), respectively.

First Round of Selection: The Threshold Mod$\boldsymbol{e l}$. To obtain knowledge on the parameter under selection $\left(1 / \gamma_{L}, 1 / \beta\right.$, or $1 / \nu$, from now on expressed as $\left.\theta\right)$, the response to selection was calculated with a threshold model assuming a standard normal distribution on an underlying liability scale. On this scale, the initial prevalence $p_{0}$ was translated to liability $x_{0}=F^{-1}\left(p_{0}\right)$, with $F^{-1}$ being the inverse standard normal density function. The first selection round results in a new liability $x_{1}=x_{0}-R$, with $R$ being the response to selection, and in a new prevalence $p_{1}=F\left(x_{1}\right)$. The response to selection was calculated as $R=\frac{i r_{I H} h \sigma_{p}}{2}$, with

- $i$ as the intensity of selection, which is equal to $\frac{d\left[F^{-1}(1-p)\right]}{1-p}$, with $d$ being the density function of the standard normal distribution. Prevalence $p$ differs for dam and sire selection. For dam selection, prevalence is defined as the prevalence of test positives for Johne's disease at the time point at which the observations are made. For sire selection, prevalence is defined as the exclusion of $20 \%$ of sires producing the most susceptible offspring based on their breeding values (constant over generations).

- $r_{I H}$ as the accuracy of selection, which is different for sire and dam selection. For dam selection, the accuracy of selection is equal to $\sqrt{h^{2}}$. For sire selection, the accuracy is dependent on the prevalence in the population as sire breeding values for resistance to Johne's disease are estimated based on test results obtained in the cow population. The accuracy was calculated as follows:

$$
r_{I H}=\sqrt{\frac{\frac{1}{4} z^{2} h^{2}}{\frac{1}{4} z^{2} h^{2}+\left[p(1-p)-\frac{1}{4} z^{2} h^{2}\right] / n}},
$$

where $p$ is the prevalence of Johne's disease in the dairy cattle population, $n$ is the average number of progeny of sire in the population (set to 100), and $z$ is equal to $d\left[F^{-1}(p)\right]$, with $d$ being the density function of the standard normal distribution.

- $h$ as the square root of the heritability, so $h=$ $\sqrt{0.10}$.

- $\sigma_{p}$ as the phenotypic standard deviation, equal to 1 by definition, because of the liability scale.

For dam selection as well as sire selection, genetic selection only takes place at the female level or at the male level. Dam or sire provides only $50 \%$ of the genetic material to the next generation and, therefore, the selection response was divided by 2 . To predict the effect of genetic selection when applying combined dam and sire selection, calculated selection responses were added.

Connecting to the Epidemiological Model. In the epidemiological model, $p_{1}$ was used to calculate $\theta_{1}$, the value for the trait under selection in the next generation. The response to selection in the first round was thus calculated as $\left|\theta_{1}-\theta_{0}\right|$, which was in turn used to calculate the phenotypic standard deviation of the trait under selection $\left(\sigma_{\theta}\right)$ with the standard selection response formula $i r_{I H} h \sigma_{\theta} / 2=\left|\theta_{1}-\theta_{0}\right|$, so that

$$
\sigma_{\theta}=2 \frac{\left|\theta_{1}-\theta_{0}\right|}{i r_{I H} h}
$$

with $i, r_{I H}$, and $h$ as described above.

Procedure For Subsequent Rounds of Selection. The value of $\theta_{1}$ (i.e., $1 / \gamma_{L}, 1 / \beta$, or $1 / \nu$ after the first round of selection) was used to simulate the spread 
of MAP with the epidemiological model by numerically solving the differential equations in Mathematica 7.0 software (Wolfram Research). It was assumed that selection took place every generation interval, being 3 yr for dam selection, and 6 yr for sire selection. The simulated prevalence $p(t)$ at the first time of selection was used to calculate a new value of $\theta_{2}$ by use of the standard selection response formula $\left(\theta_{2}-\theta_{1}=i r_{I H} h \sigma_{\theta}\right)$. With this new value of $\theta_{2}$, the numerical simulation was continued until the next round of selection at the end of another generation interval. This procedure was repeated until the proportion of subclinically and clinically infected animals in the population was less than 0.005 , representing a zero prevalence of infection in a herd of 100 cows (less than 0.5 test-positive cows).

\section{Output Evaluation}

To compare the effect of genetic selection for Johne's disease resistance between the different selection methods (dam selection, sire selection, and combined) and parameters under selection (length of latent period 1/ $\gamma_{L}$, susceptibility $1 / \beta$, and length of susceptible period $1 / \nu)$, we used the time in years to reach the proportion of subclinically and clinically infected cows of less than 0.005 , representing a zero prevalence of infection considering a herd of 100 cows. Furthermore, to measure the impact of selection for the infection itself, we calculated the change in length of latent period $\left(1 / \gamma_{L}\right)$, susceptibility $(1 / \beta)$, and length of susceptible period $(1 / \nu)$ in the first round of sire selection and at the final selection round, when the prevalence of 0.005 was reached.

We looked at 3 alternative scenarios, both as a sensitivity analysis per se and to address secondary questions:

1. a higher value of the heritability $h^{2}$ : according to Bishop et al. (2012), an imperfect diagnostic test can result in biased estimates of $h^{2}$, which can be corrected by a factor $(S p+S e-1)^{2} d\left[F^{-1}(p)\right]^{2} /$ $d\left[F^{-1}\left(p^{\prime}\right)\right]^{2}$, in which $S e$ is the sensitivity of the test, $S p$ is the specificity of the test, $p$ is the true prevalence, and $p^{\prime}=(1-S p)+(S p+S e-1) p$ is the apparent prevalence. For our estimate, an ELISA had been used with $S e=0.4$ and $S p=$ 1.0 in a subset of the whole population, with $P$ $>0.10$ (van Hulzen et al., 2011). We assumed $P$ $=0.15$ for our bias estimate, resulting in a corrected $h^{2}\left(h_{c}^{2}\right)=0.236$.

2. a higher accuracy of sire selection: to address the question in how far better estimates of sire breeding values would improve selection, we con- sidered the accuracy $r_{I H}$ to be based on a 200 head offspring rather than 100 .

3. a higher intensity of sire selection: to address the question in how far selection of a smaller proportion of sires would improve selection, we considered a selection intensity $i$ based on the best $50 \%$ rather than the best $80 \%$ of sires.

\section{RESULTS}

\section{Short-Term Decrease in Prevalence}

Figure 2 shows the decrease in prevalence with sire selection in the first $20 \mathrm{yr}$. The fastest response can be expected if the genetic variation is in the latent period $\left(1 / \gamma_{L}\right)$, but for none of the 3 parameters is a steadystate prevalence reached after $6 \mathrm{yr}$, the first generation. This has 2 consequences. First, selection of sires for the second generation is carried out with a higher prevalence $p(6 \mathrm{yr})$ than the calculated $p_{1}$ of the first selection round, based on the threshold model $\left(p_{1}=\right.$ 0.082). This accuracy of sire selection (or the intensity of dam selection) is, therefore, slightly higher than if the steady-state prevalence would have been reached. Second, the genetic progress goes faster than the infection dynamics, which causes the decrease in prevalence to lag behind the genetic improvement. This is seen in Figure 2 (and Figures 3-5, see below) as a faster decrease in prevalence after each selection round in the first couple of generations.

\section{Dam Selection Versus Sire Selection}

For dam selection (i.e., selecting those cows that test negative for Johne's disease at the time point at which the observations are made), responses to selection assuming single-trait selection for Johne's disease resistance were small, irrespective of the parameter that was affected by genetic selection (Figure 3 ). For the different parameters under selection [length of latent period $\left(1 / \gamma_{L}\right)$, susceptibility $(1 / \beta)$, and length of susceptible period $(1 / \nu)], 702,486$, and $379 \mathrm{yr}$ were needed to reach a 0.005 prevalence of infection, respectively.

For sire selection (i.e., selecting $80 \%$ of sires producing the least susceptible offspring based on their breeding values), responses to selection for Johne's disease resistance were larger compared with dam selection (Figure 4). As for dam selection, it did matter which parameter was under selection to reach a 0.005 prevalence of infection. Length of latent period $\left(1 / \gamma_{L}\right)$, susceptibility $(1 / \beta)$, and length of susceptible period $(1 / \nu)$ required 223,179 , and 147 yr to reach a 0.005 prevalence of infection, respectively.

Combined selection resulted in the largest response to selection for Johne's disease resistance (Figure 5). 


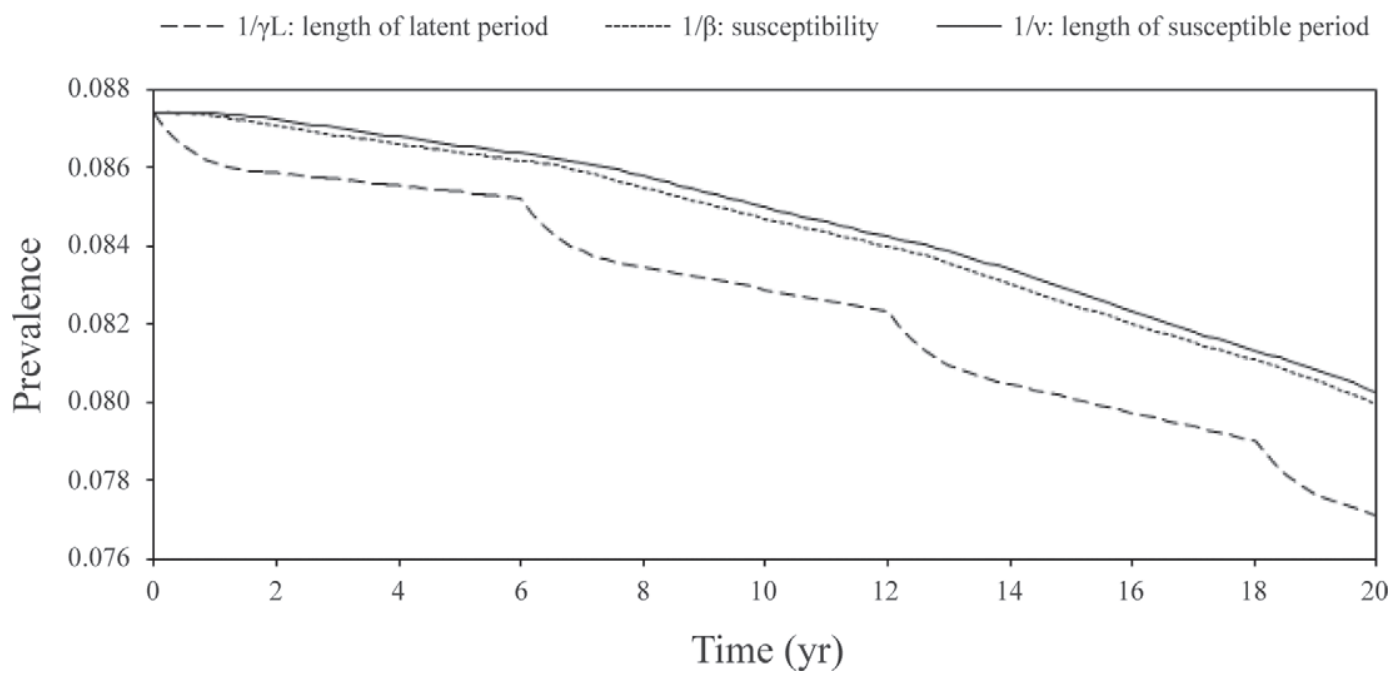

Figure 2. First $20 \mathrm{yr}$ of predicted response to selection for resistance to Johne's disease when selecting $80 \%$ of sires producing the least susceptible offspring based on their breeding values for the 3 parameters under selection $\left(1 / \gamma_{L}, 1 / \beta\right.$, or $\left.1 / \nu\right)$. The effect of selection on the parameters under selection was translated to an effect on prevalence (y-axis) to assess the contribution of genetic selection to control of Johne's disease.

Depending on the parameter under selection, length of latent period $\left(1 / \gamma_{L}\right)$, susceptibility $(1 / \beta)$ and length of susceptible period $(1 / \nu)$, respectively 185,148 , and 120 yr were needed to reach a 0.005 prevalence of infection.

\section{Parameters Under Selection}

Response to selection assuming single-trait selection for Johne's disease resistance was largest for the length of the susceptible period $(1 / \nu)$, followed by the susceptibility $(1 / \beta)$, and then the length of the latent period $\left(1 / \gamma_{L}\right)$, irrespective of the selection method applied.
Table 2 shows change in parameters under selection after 1 round of sire selection and after the final round of sire selection needed to reach a 0.005 prevalence of infection. The length of the latent period increased from 50 to 52.4 wk $(+4.9 \%)$ after 1 round of selection on $1 / \gamma_{L}$, the number of infectious doses needed to become infected increased from 5.71 to 5.79 doses $(+1.2 \%)$ after 1 round of selection on $1 / \beta$, and the length of the susceptible period decreased from 50 to $49.3 \mathrm{wk}(-1.5 \%)$ after 1 round of selection on $1 / \nu$. At the time of reaching the final prevalence of 0.005 , the latent period $1 / \gamma_{L}$ had increased from 1 to almost 2.5 yr, susceptibility $(1 / \beta)$ from 5.71 to 7.44 doses required

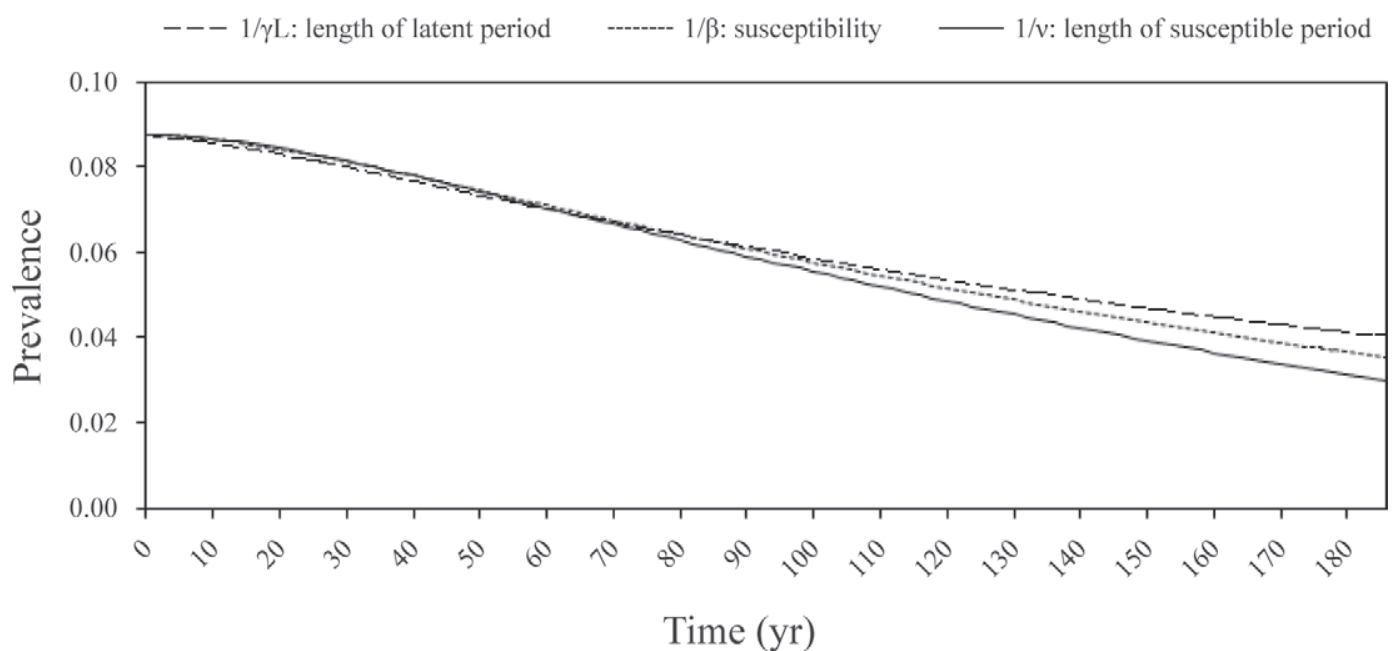

Figure 3. Predicted response to selection for resistance to Johne's disease when selecting those animals that do not show clinical signs at the time point at which the observations are made for the 3 parameters under selection $\left(1 / \gamma_{L}, 1 / \beta\right.$, or $\left.1 / \nu\right)$. The effect of selection on the parameters under selection was translated to an effect on prevalence (y-axis) to assess the contribution of genetic selection to control of Johne's disease. 


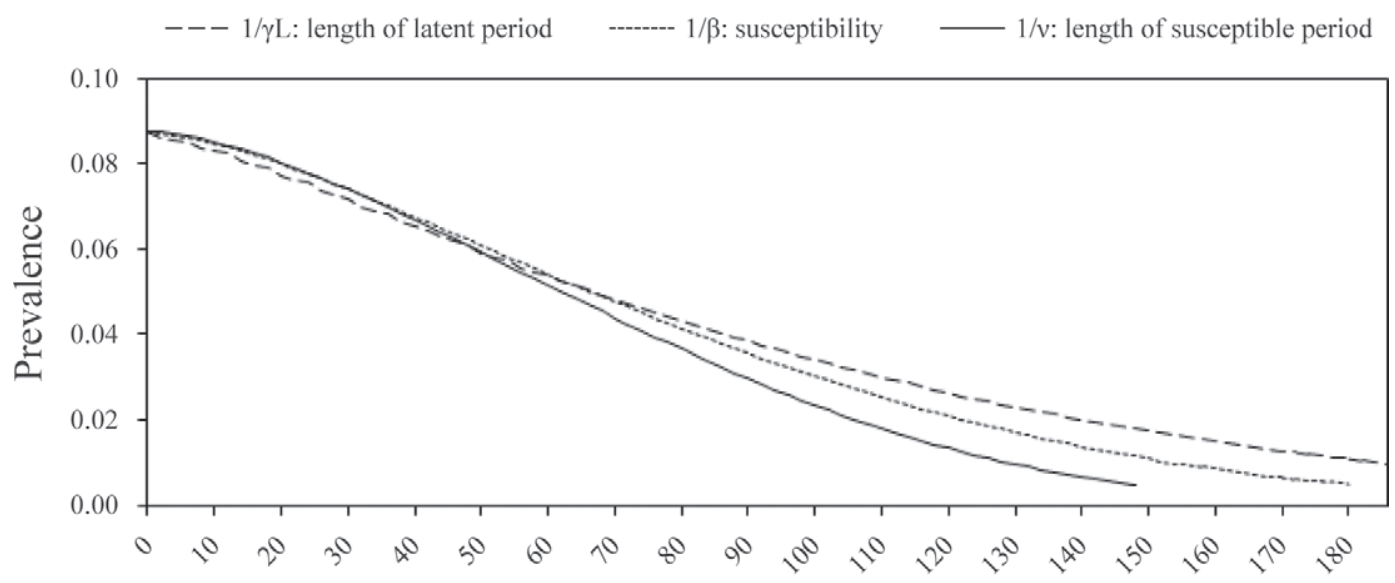

Time $(\mathrm{yr})$

Figure 4. Predicted response to selection for resistance to Johne's disease when selecting $80 \%$ of sires producing the least-susceptible offspring based on their breeding values for the 3 parameters under selection $\left(1 / \gamma_{L}, 1 / \beta\right.$, or $\left.1 / \nu\right)$. The effect of selection on the parameters under selection was translated to an effect on prevalence (y-axis) to assess the contribution of genetic selection to control of Johne's disease.

for infection, and the latent period $(1 / \nu)$ had decreased from 1 to 0.69 yr.

\section{Sensitivity Analysis}

The sensitivity analyses for sire selection are shown in Table 3, and they are not qualitatively different for dam selection or combined selection. Correcting our heritability estimate for bias does improve selection results, but not sufficiently to reach the 0.005 prevalence within a human lifetime. The same holds for increasing accuracy or intensity of selection.

\section{DISCUSSION}

The objective of this study was to model genetic selection for Johne's disease resistance and to study the effect of different selection strategies on the prevalence in the dairy cattle population. Selection of those cows that test negative for Johne's disease at the time point at which the observations are made (dam selection), achieved the smallest response to selection. Based on these results, the genetic response resulting from the certification-and-surveillance program currently used in the Netherlands will not substantially contribute to Johne's disease elimination in the Netherlands. Selec-

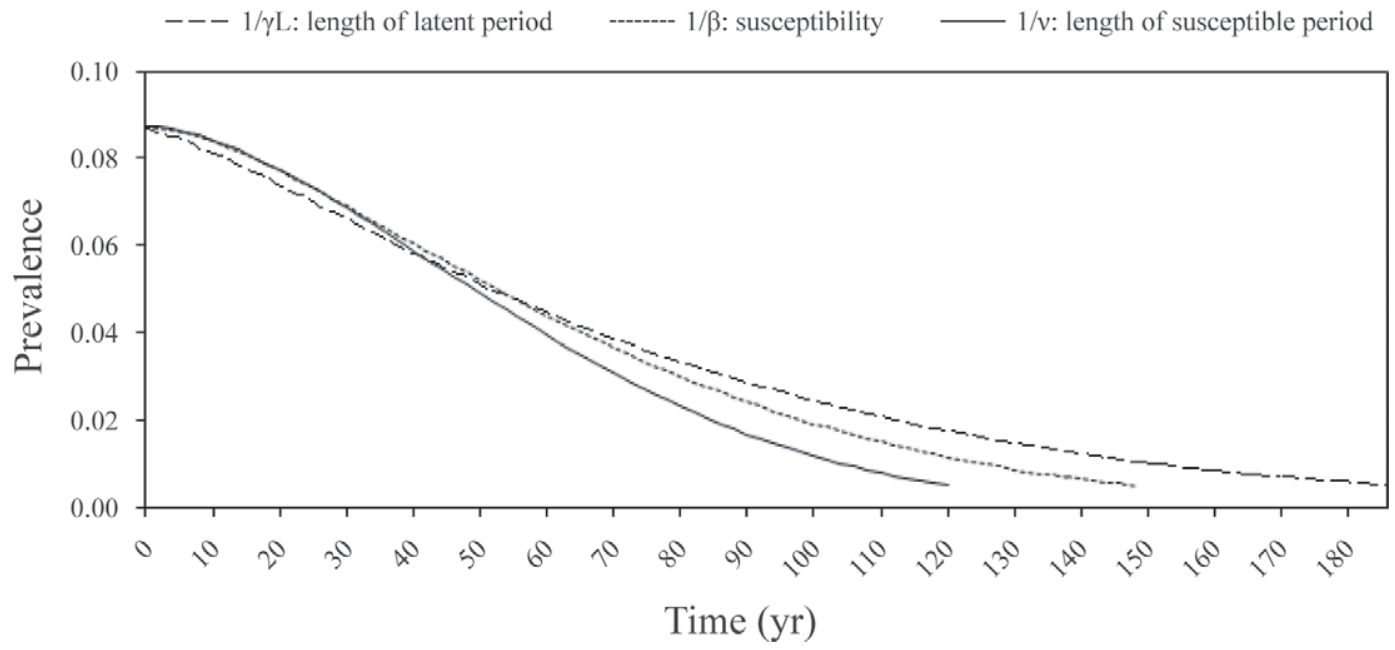

Figure 5. Predicted response to selection for resistance to Johne's disease when selecting those animals that do not show clinical signs at the time point at which the observations are made and $80 \%$ of sires producing the least-susceptible offspring based on their breeding values for the 3 parameters under selection $\left(1 / \gamma_{L}, 1 / \beta\right.$, or $\left.1 / \nu\right)$. The effect of selection on the parameters under selection was translated to an effect on prevalence (y-axis) to assess the contribution of genetic selection to control of Johne's disease. 
Table 2. Results for sire selection: change in parameters under selection after 1 round of genetic selection and after the final round of selection ${ }^{1}$

\begin{tabular}{lccc}
\hline & \multicolumn{3}{c}{ Parameter under selection } \\
\cline { 2 - 4 } Item & $1 / \gamma_{L}$ & $1 / \beta$ & $1 / \nu$ \\
\hline Start value & 50 & 5.71 & 50 \\
Value after first round of selection & $52.4(+4.9 \%)$ & $5.79(+1.2 \%)$ & $49.3(-1.5 \%)$ \\
Value after final round of selection & $121.6(+143 \%)$ & $7.44(+30 \%)$ & $34.7(-31 \%)$ \\
Required time (yr) & 223 & 179 & 147 \\
Required number of generations & 37 & 29 & 24 \\
\hline${ }^{1}$ Relative change (expressed in percentages in comparison with start value) is shown in parentheses. Also shown \\
are the time and number of generations it takes to reach a prevalence of 0.005 . Parameters under selection are \\
length of latent period in weeks $\left(1 / \gamma_{L}\right)$, susceptibility $(1 / \beta)$, and length of susceptible period in weeks $(1 / \nu)$.
\end{tabular}

tion of $80 \%$ of sires producing the least-susceptible offspring based on their breeding values (sire selection) resulted in larger response to selection compared with dam selection. Although the number of generations that is needed to reach a 0.005 prevalence of infection remains substantial, sire selection can contribute to control of Johne's disease in the long run. As expected, sensitivity analysis for selection intensity shows that an increase in selection intensity to $50 \%$ results in an increase in selection response. However, we feel that a selection intensity of $80 \%$ is more realistic, as focus for genetic improvement is still primarily on production traits. Increasing the accuracy (based on 200 rather than 100 daughters) also increases the selection response, but this effect is relatively small and may even disappear when taking into account the resulting increase in generation time.

The large difference in selection response between dam selection and sire selection was mainly due to differences in accuracy of selection and selection intensity. Dam selection, as used in this study, only used information on the cow itself (own performance) to make selection decisions. Consequently, the accuracy of selection was low. In this study, sire selection had a much higher accuracy because information from 100 daughters was used to estimate a breeding value for Johne's disease resistance. Also, selection intensity as applied in this study was larger for sire selection (i.e., $80 \%$ of sires producing the least-susceptible offspring based on their breeding values were selected to produce animals for the next generation). Selection intensity when applying dam selection was equal to 100 minus the prevalence of Johne's disease in the dairy population and had a minimum value of $91.3 \%$.

For dam selection as well as for sire selection, nonlinearity in the response in prevalence to selection is caused by a decrease in prevalence. For dam selection, the decrease in prevalence also affected the intensity of selection because the selected proportion of animals in the population was equal to $1-p$. For sire selection, the decrease in prevalence of disease reduced the accuracy of selection, as it was dependent on the prevalence of Johne's disease in the population.

Earlier research showed that genetic variation for resistance to Johne's disease exists (Koets et al., 2000; Mortensen et al., 2004; Gonda et al., 2006; Hinger et al., 2007; Attalla et al., 2010; van Hulzen et al., 2011; Küpper et al., 2012). However, knowledge of genes and biological pathways involved in resistance to Johne's disease is still incomplete. Therefore, all parameters included in the model were considered for their potential involvement in the existence of genetic differences between cows in resistance to Johne's disease in this study. To our knowledge, only the length of the latent period, susceptibility, and the length of the susceptible period may be involved in the existence of genetic dif-

Table 3. Sensitivity analysis for sire selection: required time (yr) to reach a prevalence of $0.005^{1}$

\begin{tabular}{lcrr}
\hline & \multicolumn{3}{c}{ Parameter under selection } \\
\cline { 2 - 4 } Item & $1 / \gamma_{L}$ & $1 / \beta$ & $1 / \nu$ \\
\hline Default model & 223 & 179 & 147 \\
Corrected $h^{2}=0.236$ & 139 & 115 & 94 \\
Accuracy $r_{I H}$ based on offspring size of 200 & 192 & 157 & 128 \\
Intensity $i$ based on selecting best $50 \%$ & 126 & 105 & 85 \\
\hline${ }^{1}$ In the default model, the initial prevalence is 0.087, selection is continued until the prevalence is 0.005 , herita- \\
bility $\left(h^{2}\right)=0.1, r_{I H}$ is accuracy based on offspring size of 100 , and intensity $(i)$ is based on a selection intensity \\
of $80 \%$ best sires. Parameters under selection are length of latent period in weeks $\left(1 / \gamma_{L}\right)$, susceptibility $(1 / \beta)$, \\
and length of susceptible period in weeks $(1 / \nu)$.
\end{tabular}


ferences between cows. Another important infection characteristic that can be different between animals is the rate of shedding of bacteria (Nieuwhof et al., 2009; Doeschl-Wilson et al., 2011). However, this will not affect the chance of a calf to become infected and to progress to the shedding and eventually clinical phase. To achieve lower shedding levels, selection should take place on the shedding level itself.

If genetic variation for resistance to Johne's disease has an impact on the length of the latent period $\left(1 / \gamma_{L}\right)$, response to selection will be slower compared with the susceptibility $(1 / \beta)$ and the length of the susceptible period $(1 / \nu)$. As only infected animals were affected by selection on the length of the latent period, the value of this parameter influences only a minor part of the population. All animals except the calves infected through vertical transmission reside in the susceptible compartment for the initial period of their lives and, therefore, selection on susceptibility $(1 / \beta)$ and the length of the susceptible period $(1 / \nu)$ influence a major part of the population. Additional analysis showed that the ranking of parameters under selection was not sensitive to disease prevalence in the population that was twice as high and twice as low compared with the initial prevalence used in this study (results not shown).

Three particular aspects of combining genetic and epidemiological models require special attention and should be investigated theoretically in more depth. First, in our epidemiological model, animals in a particular compartment $(S)$ all had the same rate at which they were culled $(\mu)$ or progressed to the next compartment $(T$ or $R$ ). The assumption of a homogenous group of animals within each compartment is in conflict with the existence of genetic variability among cows. The model should ideally include between-animal variation in the rate parameters that describe the transmission of infection, the progress to the resistant, or the progress from the latent to the shedding compartment.

Second, the prevalence of the infection had not reached an endemic steady state at each selection round. We used the actual prevalence at the time of selection in the selection model to determine the value of $\theta$ in the next generation. The selection model assumes that the heritability remains the constant over subsequent generations, which means that the relative contributions of the additive genetic variation and environmental variation to the infection status of animals remains the same. It is not clear if this assumption is still valid if the population has not yet reached a new steady-state prevalence.

Third, it is assumed that selection takes place in a single population with a starting prevalence of 0.087 , which was used to find the value for $\beta$ by calibration. In reality, some herds have higher and others have lower prevalence, based on herd-related factors such as housing and management. If selection of sires on a national level results in improvement such that the average herd loses MAP, this may not be sufficient for the herds with initially higher prevalence. On the other hand, the existence of such herds may improve sire selection for the population as a whole, as they provide environments in which differences between cows are still observed at a time when the national prevalence is very low.

Two other features of the model may cause overestimation of the response to selection. First, animals that do not become infected during early age are assumed to gain long-lasting immunity may not be correct. In general, the view that older animals are less susceptible to Johne's disease is quite accepted but the assumption of long-lasting immunity may be too strict. If the susceptible period is longer than modeled in this study and (or) animals first show a decrease in susceptibility before gaining long-lasting immunity, response to selection is likely to be different from results in this study. Second, the test used to distinguish the infected from the noninfected animals is assumed to be a perfect test (able to detect all subclinically and clinically infected cows in the population). In reality, the sensitivity of tests used to detect Johne's disease are low and the tests are only able to find only a fraction of the subclinically and clinically infected cows in the population, which will also result in a slower response to selection.

In relation to our conclusion that sire selection can contribute to control of Johne's disease in the long run, we also searched in the literature for the effect of other control strategies. In the current study, we used a reduced version of the model of Marcé et al. (2011a), who applied the model to consider the effect of decreasing exposure of calves to different contaminated environments on the prevalence of infectious adults, specifically through the use of different housing facilities, cleaning, and culling clinically infected cows. However, comparison of their and our results is complicated, because they looked at establishment of the infection in a previously unaffected herd, whereas we started our simulations in an endemically infected population. The study of Collins and Morgan (1991) showed that, in agreement with other studies done in earlier years (Ringdal, 1965; Julian, 1975; Moyle, 1975), the control of Johne's disease in a dairy herd takes many years. A simulation model is described predicting the rate at which Johne's disease can be controlled or eliminated on the level of a dairy herd, incorporating parameters describing the accuracy of the diagnostic test, the number of calf-cow contacts resulting from improved hygiene or vaccination, the prevalence of disease in the herd when the control program was initiated, and whether the herd was open or closed. Of those parameters, only tests 
with a sensitivity of at least $70 \%$ were able to reduce the prevalence of Johne's disease to less than $1 \%$ in less than 10 yr. However, even today, diagnosis is hampered by imperfect tests. Test sensitivity of an ELISA, most commonly used because of its simplicity and low cost, is, on average, around 40\%. In addition, other studies showed that efficient closure of transmission routes is necessary for control (Groenendaal et al., 2002; Dorshorst et al., 2006; Kudahl et al., 2007, 2008). And efficient control measures often involve labor-intensive procedures that may be difficult to apply on busy days. For those reasons, we suggest applying a combination of strategies, including genetic selection on the sire level, to become as efficient as possible in controlling Johne's disease.

\section{CONCLUSIONS}

The aim of this study was to model the effect of genetic selection for resistance on Johne's disease resistance and to study the effect of different selection strategies on Johne's disease prevalence in the dairy cattle population. Based on the assumed model and parameters under selection, we can draw the following conclusions. First, the genetic effect of the current certification-and-surveillance program in the Netherlands is small. Second, sire selection is able to contribute to control of Johne's disease in the long run. Such a strategy should be used in combination with other control strategies, which are also essential for the collection of information for the breeding program.

\section{ACKNOWLEDGMENTS}

The authors thank CRV (Arnhem, the Netherlands) for facilitating previous research on genetic variation in susceptibility in dairy cattle that forms a fundament for this manuscript. The authors also thank S. C. Bishop [The Roslin Institute and Royal (Dick) School of Veterinary Studies, Edinburgh, UK] for advice and P. Bijma (Animal Breeding and Genomics Centre, Wageningen, the Netherlands) for his valuable contribution to a part of the model analysis.

\section{REFERENCES}

Attalla, S. A., A. J. Seykora, J. B. Cole, and B. J. Heins. 2010. Genetic parameters of milk ELISA scores for Johne's disease. J. Dairy Sci. 93:1729-1735.

Bastida, F., and R. A. Juste. 2011. Paratuberculosis control: A review with a focus on vaccination. J. Immune Based Ther. Vaccines 9:8.

Benedictus, A., R. M. Mitchell, M. Linde-Widmann, R. Sweeney, T. Fyock, Y. H. Schukken, and R. H. Whitlock. 2008. Transmission parameters of Mycobacterium avium subspecies paratuberculosis infections in a dairy herd going through a control program. Prev. Vet. Med. 83:215-227.
Bishop, S. C., A. B. Doeschl-Wilson, and J. A. Woolliams. 2012. Uses and implications of field disease data for livestock genomic and genetics studies. Front. Genet. 3:114.

Collins, M. T., and I. R. Morgan. 1991. Economic decision analysis model of a paratuberculosis test and cull program. J. Am. Vet. Med. Assoc. 199:1724-1729.

Doeschl-Wilson, A. B., R. Davidson, J. Conington, T. Roughsedge, M. R. Hutchings, and B. Villanueva. 2011. Implications of host genetic variation on the risk and prevalence of infectious diseases transmitted through the environment. Genetics 188:683-693.

Dorshorst, N. C., M. T. Collins, and J. E. Lombard. 2006. Decision analysis model for paratuberculosis control in commercial dairy herds. Prev. Vet. Med. 75:92-122.

Gonda, M. G., Y. M. Chang, G. E. Shook, M. T. Collins, and B. W. Kirkpatrick. 2006. Genetic variation of Mycobacterium avium ssp. paratuberculosis infection in US Holsteins. J. Dairy Sci. 89:18041812.

Groenendaal, H., M. Nielen, A. W. Jalvingh, S. H. Horst, D. T. Galligan, and J. W. Hesselink. 2002. A simulation of Johne's disease control. Prev. Vet. Med. 54:225-245.

Hill, W. G. 2010. Understanding and using quantitative genetic variation. Phil. Trans. R. Soc. 365:73-85. http://dx.doi.org/10.1098/ rstb.2009.0203.

Hinger, M., H. Brandt, S. Horner, and G. Erhardt. 2007. Short communication: Association analysis of microsatellites and Mycobacterium avium subspecies paratuberculosis antibody response in German Holsteins. J. Dairy Sci. 90:1957-1961.

Jorgensen, J. B. 1977. Survival of Mycobacterium paratuberculosis in slurry. Nord. Vet. Med. 29:267-270.

Jørgensen, J. B. 1982. An improved medium for culture of Mycobacterium paratuberculosis from bovine faeces. Acta Vet. Scand. 23:325-335.

Julian, R. J. 1975. A short review and some observations on Johne's disease with recommendations for control. Can. Vet. J. 16:33-43.

Koets, A. P., G. Adugna, L. L. Janss, H. J. van Weering, C. H. J. Kalis, G. H. Wentink, V. P. M. G. Rutten, and Y. H. Schukken. 2000. Genetic variation of susceptibility to Mycobacterium avium subsp. paratuberculosis infection in dairy cattle. J. Dairy Sci. 83:2702-2708.

Kudahl, A. B., S. S. Nielsen, and S. Østergaard. 2008. Economy, efficacy, and feasibility of a risk-based control program against paratuberculosis. J. Dairy Sci. 91:4599-4609.

Kudahl, A. B., S. Østergaard, J. T. Sørensen, and S. S. Nielsen. 2007. A stochastic model simulating paratuberculosis in a dairy herd. Prev. Vet. Med. 78:97-117.

Küpper, J., H. Brandt, K. Donat, and G. Erhardt. 2012. Heritability estimates for Mycobacterium avium subspecies paratuberculosis status of German Holstein cows tested by fecal culture. J. Dairy Sci. 95:2734-2739.

Marcé, C., P. Ezanno, H. Seegers, D. U. Pfeiffer, and C. Fourichon. 2011a. Within-herd contact structure and transmission of Mycobacterium avium subspecies paratuberculosis in a persistently infected dairy cattle herd. Prev. Vet. Med. 100:116-125.

Marcé, C., P. Ezanno, H. Seegers, D. U. Pfeiffer, and C. Fourichon. 2011b. Predicting fadeout versus persistence of paratuberculosis in a dairy cattle herd for management and control purposes: A modelling study. Vet. Res. 42:36.

Matthews, H. T. 1947. On Johne's disease. Vet. Rec. 59:397-401.

Mortensen, H., S. S. Nielsen, and P. Berg. 2004. Genetic variation and heritability of the antibody response to Mycobacterium avium subspecies paratuberculosis in Danish Holstein cows. J. Dairy Sci. 87:2108-2113.

Moyle, A. I. 1975. Culture and cull procedure for control of paratuberculosis. J. Am. Vet. Med. Assoc. 166:689-690.

Nielsen, S. S. 2008. Transitions in diagnostic tests used for detection of Mycobacterium avium subsp. paratuberculosis infections in cattle. Vet. Microbiol. 132:274-282.

Nielsen, S. S. 2009. Programmes on paratuberculosis in Europe. Pages 101-108 in Proc. 10th International Colloquium on Paratuberculosis, Minneapolis, MN. Accessed Jan. 13, 2014. http://www.paratuberculosis.info/images/stories/pdfs/907-506-1-PB.pdf. 
Nielsen, S. S., and A. K. Ersbøll. 2006. Age at occurrence of Mycobacterium avium subspecies paratuberculosis in naturally infected dairy cows. J. Dairy Sci. 89:4557-4566.

Nieuwhof, G. J., J. Conington, and S. C. Bishop. 2009. A genetic-epidemiological model to describe resistance to an endemic bacterial disease in livestock: Application to footrot in sheep. Genet. Sel. Evol. http://dx.doi.org/10.1186/1297-9686-41-19.

Ringdal, G. 1965. Studies on Johne's disease in a single herd during a five-year period. Nord. Vet. Med. 17:73-96.

Rossiter, C. A., and W. S. Burhans. 1996. Farm-specific approach to paratuberculosis (Johne's disease) control. Vet. Clin. North Am. Food Anim. Pract. 12:383-415.

Stabel, J. R. 1998. Johne's disease: A hidden threat. J. Dairy Sci. 81:283-288.

van Hulzen, K. J. E., M. Nielen, A. P. Koets, G. de Jong, J. A. M. van Arendonk, and H. C. M. Heuven. 2011. Effect of herd prevalence on heritability estimates of antibody response to Mycobacterium avium subspecies paratuberculosis. J. Dairy Sci. 94:992-997.

van Roermund, H. J. W., D. Bakker, P. T. J. Willemsen, and M. C. M. de Jong. 2007. Horizontal transmission of Mycobacterium avium subsp. paratuberculosis in cattle in an experimental setting: Calves can transmit the infection to other calves. Vet. Microbiol. 122:270-279.

Whitlock, R. H., S. J. Wells, R. W. Sweeney, and J. Van Tiem. 2000. ELISA and fecal culture for paratuberculosis (Johne's disease): Sensitivity and specificity of each method. Vet. Microbiol. 77:387-398.

Whittington, R. J., D. J. Marshall, P. J. Nicholls, I. B. Marsh, and L. A. Reddacliff. 2004. Survival and dormancy of Mycobacterium avium subsp. paratuberculosis in the environment. Appl. Environ. Microbiol. 70:2989-3004.

Whittington, R. J., I. A. Reddacliff, I. B. Marsh, S. McAllistair, and V. Saunders. 2000. Temporal patterns and quantification of excretion of Mycobacterium avium subsp paratuberculosis in sheep with Johne's disease. Aust. Vet. J. 78:34-37.

Whittington, R. J., and P. A. Windsor. 2009. In utero infection of cattle with Mycobacterium avium subsp. paratuberculosis: A critical review and meta-analysis. Vet. J. 179:60-69. 